\title{
OBSERVATORIO
}

\section{INVESTIGAR EL PERIODISMO DESDE LA PERSPECTIVA DE LAS AUDIENCIAS}

\author{
Investigating journalism from the perspective \\ of the audience
}

\section{Pere Masip}

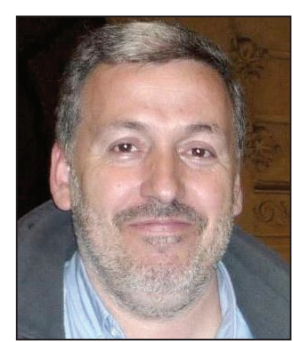

Pere Masip, profesor titular de periodismo de la Universidad Ramon Llull, es el investigador principal del grupo de investigación Digilab: media, strategy and regulation. Sus principales áreas de interés son el periodismo digital, la convergencia mediática y el impacto de la tecnología en las prácticas periodísticas y comunicativas. Coordina el proyecto financiado por el Ministerio de Economía y Competitividad Audiencias activas y establecimiento de la agenda en la esfera pública digital y la edición española del Media pluralism monitor, financiado por la Comisión Europea. Es autor de múltiples artículos y libros.

https://orcid.org/0000-0002-8231-0824

Facultat de Comunicació i Relacions Internacionals Blanquerna. Universitat Ramon Llull Plaça de Joan Coromines, s/n. 08001 Barcelona, España peremm@blanquerna.url.edu

\section{Resumen}

En el actual sistema mediático, la relación entre los medios y la audiencia ha sufrido una importante mutación. Una cuestión que no es trivial en la medida que la audiencia es imprescindible para que exista periodismo. Paradójicamente, sin embargo, los medios han prestado poca atención a la audiencia, más allá de la proporcionada por las métricas. Desde la academia han proliferado investigaciones que han profundizado en el estudio de esa relación, pero fundamentalmente desde la perspectiva de los medios y los periodistas. En este texto se apuntan algunas líneas de investigación que abordan la relación entre audiencia y los medios, los periodistas y la información, desde la perspectiva de los usuarios.

\section{Palabras clave}

Audiencias; Audiencias activas; Periodismo; Medios; Medios digitales; Investigación.

\begin{abstract}
In the current media system, the relationship between media and audience has undergone a major mutation. This is an issue that is not trivial because the audience is essential to journalisms' existence. Paradoxically, however, the media has paid little attention to audience, beyond data provided by online metrics. However, research from the academy has proliferated and delved into the study of this relationship, but primarily from the perspective of media and journalists. In this text we investigate the relationship between audience and media, journalists and information, all from the users' perspective.
\end{abstract}

\section{Keywords}

Audiences; Active audiences; Journalism; Media; Digital media; Research.

Masip, Pere (2016). "Investigar el periodismo desde la perspectiva de las audiencias". El profesional de la información, v. 25, n. 3 , pp. $323-330$.

http://dx.doi.org/10.3145/epi.2016.may.01

\section{Introducción ${ }^{1}$}

En los últimos 15 años, la influencia de factores tecnológicos, organizacionales, profesionales, económicos, sociales y culturales ha conducido a la configuración de un sistema mediático mucho más complejo. La distinción entre nuevos y viejos medios pierde sentido, unos y otros se hibridan, incorporan posibilidades preexistentes, las transforman y de- sarrollan otras nuevas. El propio rol del periodismo debe ser reformulado (Chadwick, 2013).

Durante este tiempo han proliferado nuevos actores (periodistas ciudadanos, activistas, relaciones públicas, agregadores, buscadores, redes sociales, etc.) y las relaciones entre ellos se han intensificado y diversificado. En este sentido, uno de los cambios más relevantes ha sido que el periodis- 


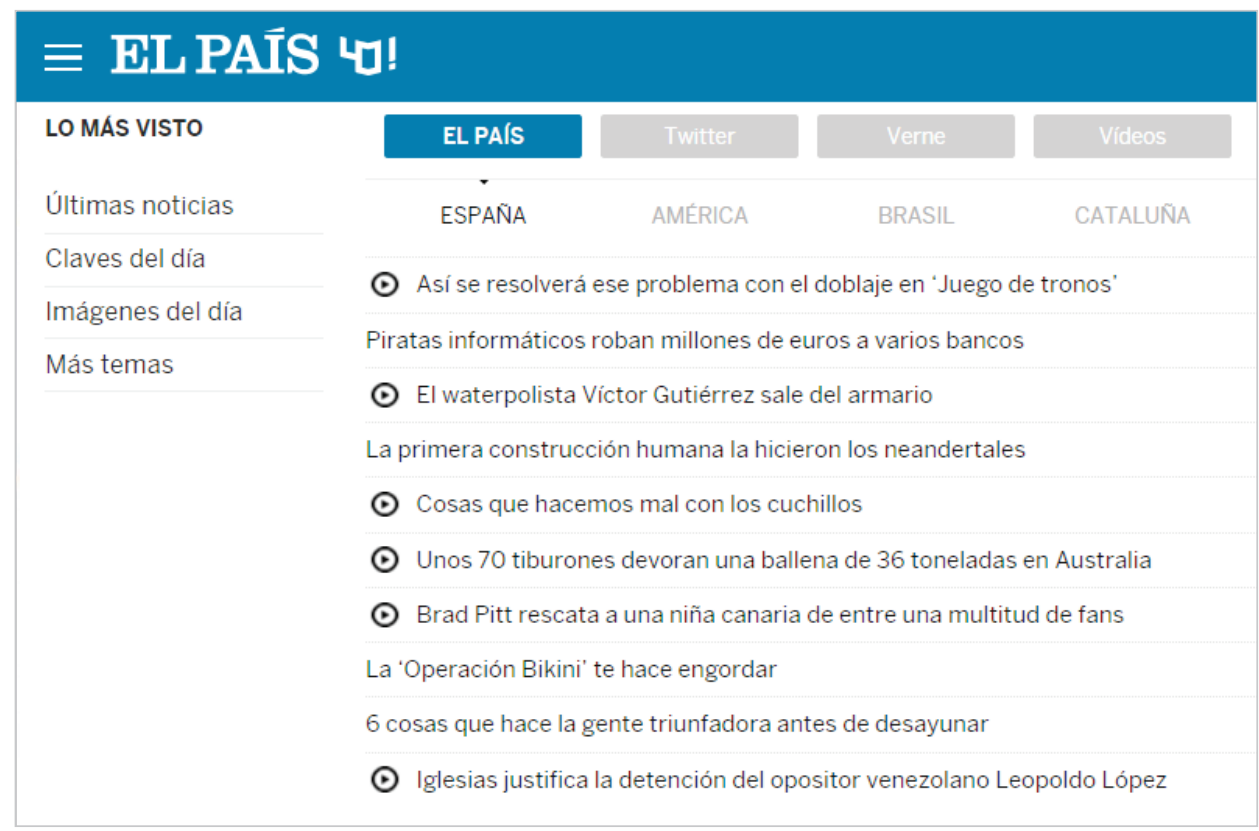

Figura 1. Noticias más vistas en El país http://elpais.com/lomasvisto/index.htm/

mo ha salido de las redacciones, a pesar de que, como veremos, los medios continúan ejerciendo un papel central en el nuevo ecosistema informativo.

A lo largo de este proceso de reconfiguración, los medios tradicionales han visto como su credibilidad se ha puesto en entredicho (Newman; Levy; Nielsen, 2015; Suau, 2015). Los ciudadanos los perciben como partidistas e interesados en mantener una agenda propia marcada por sus estrechos vínculos con los poderes públicos y económicos (Ruiz-Caballero, 2008). A estos asedios, como los denomina RuizCaballero, se les superpone una crítica situación económica, con audiencias cada vez menores y una crisis en el modelo de negocio que ha conducido a una progresiva disminución de ingresos. De manera constante se ha producido la reducción de plantillas ${ }^{2}$ y una uniformidad de los contenidos, un periodismo low cost basado en el copy and paste (Fenton, 2010). La homogenización de los contenidos se agudiza con la monitorización de la competencia, que permite reducir riesgos en la toma de decisiones (Boczkowski, 2010), y su canibalización (Phillips, 2011), es decir, evitar la atribución de las noticias producidas por los competidores ${ }^{3}$.

La marca del medio pierde valor, ya no es importante qué se dice, ni quién lo dice

Los medios han desatendido a sus lectores, ha arraigado el cortar y pegar y el trending topic. La información se ha convertido en una commodity, un producto para satisfacer las necesidades inmediatas (léase visitas o likes), y, por tanto, un producto fácilmente reemplazable. La marca pierde valor, ya no es importante qué se dice, ni quién lo dice. Los periódicos se han convertido en vendedores de servicios publicitarios y no de contenidos (Sáez, 2015).
En este contexto, la relación entre el periodismo y su audiencia ha sufrido una importante transformación. Una cuestión, ésta, que no es trivial en la medida que la audiencia es imprescindible para que exista periodismo. Mientras que no se ha dudado en anunciar la desaparición de los periódicos (Sabadin, 2007; Nerone, 2009; Jarvis, 2015) y la disolución del papel de los periodistas (Gillmor, 2007; Bruns, 2005), todavía nadie se ha pronunciado sobre la desaparición de la audiencia. Y no se producirá en la medida que es condición sine qua non para que exista el periodismo. Sin audiencia, no hay periodismo. La audiencia es importante desde un punto de vista normativo, el propósito del periodismo es proporcionar al ciudadano la información necesaria para ser libre y capaz de gobernarse a sí mismo (Kovach; Rosenstiel, 2003); pero lo es también desde un punto de vista estrictamente económico. Paradójicamente, sin embargo, en los últimos años los medios han sistemáticamente desoído a la audiencia, entendida como lectores ciudadanos, no como clientes soberanos.

La mayoría de medidas tomadas por los medios han sido dirigidas a retener a la audiencia por la vía del entretenimiento, pero pocas concebidas para recuperar su confianza

La relación entre periodistas y audiencia se ha basado en un pacto de confianza mutua, de expectativas compartidas, en una especie de contrato social (Karlsson; Clerwall; Nord, 2015). La confianza que los lectores depositan en los periodistas, esperando que lo que publican sea realmente lo que deberían publicar y lo que les interesará; y la confianza de los periodistas en que lo que publican sea realmente lo que el público espera recibir, y satisfaga sus expectativas como ciudadanos (Ward, 2005; Masip, 2014). Como hemos visto, esta confianza se ha desquebrajado en los últimos años.

Ante la sangría de lectores y la pérdida de ingresos los medios han tomado diversas medidas, algunas desesperadas y la mayoría dirigidas a retener a la audiencia por la vía del entretenimiento, pero pocas concebidas para atraerla a través de recuperar su confianza.

Para evitar la huida, los medios han potenciado la participación, por motivos económicos (Vujnovic et al., 2009), y 
han tendido a buscar soluciones de carácter tecnológico (Lee; Chyi, 2013), pero parecen prestar todavía poca atención a los ciudadanos, más allá de los datos que les ofrece la analítica web, que no son pocos. La progresiva reducción de la audiencia a datos cuantitativos incide en los cimientos de la cultura periodística y en la toma de decisiones de los propios periodistas (Anderson, 2011a). Fruto de esta nueva relación con la audiencia, mediatizada por las métricas, en el juicio para determinar qué es noticia y qué no lo es cada vez tienen mayor importancia los deseos de los lectores. Se pasa del interés público al interés del público.

Hay que abandonar los espacios comunes de confort de periodistas y académicos y repreguntarse las propias bases del periodismo, como ¿qué es el periodismo? o ¿qué es noticia hoy?

En los últimos años se ha dedicado especial interés a analizar la relación entre audiencias y medios, y muy en particular al papel de las audiencias activas y del periodismo participativo, percibido como una forma de establecer una nueva relación entre los periodistas y su audiencia. La mayor parte de esas investigaciones se han centrado en analizar el fenómeno desde la perspectiva de los medios y de los informadores. Son numerosos los trabajos que han analizado los mecanismos de participación impulsados por los medios (Hermida; Thurman, 2008; Singer et al., 2011; Suau, 2015), han monitorizado el uso que éstos realizan de las redes sociales (García-De-Torres et al., 2011), o han indagado sobre la relación de los periodistas con los lectores y su opinión sobre la audiencia participativa (Heise; Loosen; Reimer; Schmidt, 2013).

Esos trabajos han evidenciado la actitud ambivalente de los periodistas (Borger et al., 2013), que por un lado valoran las aportaciones que pueda realizar la audiencia como fuente de información (Harrison, 2010; Robinson, 2009; Singer, 2010), pero al mismo tiempo desconfían de ella ante el temor de alterar el sistema de relaciones existente, basado en una determinada relación de poder entre los distintos actores (Singer et al. 2011, Netzer et al., 2014).

Autores como Anderson (2011a) también han identificado un doble discurso de directivos y reporteros en torno a la audiencia. Mientras que los primeros tienden a sobrevalo- rar la creatividad y la voluntad participativa de la audiencia, los segundos, mantienen con ella una relación mucho más conflictiva. Es percibida como una masa abstracta, como una multitud distante que busca información sensacionalista y superficial (Costera-Meijer, 2013), como una ignorante consumidora de contenido (Anderson, 2011b).

El desarrollo de muchas de esas investigaciones sobre las audiencias participativas ha permitido poner en evidencia que algunos de los enfoques teóricos sobre las que se sustentaban se basaban en sobrentendidos y buenos deseos. Así, desde las perspectivas normativas, la participación siempre ha sido considerada como algo positivo y deseable (Borger et al., 2013; Curran; Fenton; Freedman, 2012; Klinger; Svensson, 2015). Del mismo modo, se ha dado por sentado que los ciudadanos quieren participar, quieren producir contenidos y compartirlos con los periodistas (Jenkins; Carpentier, 2013). Sin embargo, diversas investigaciones han mostrado que las audiencias son menos activas de los imaginado (Purcell et al., 2010; Bergström, 2008; Van-Dijk, 2009; Suau, 2015; Masip et al., 2015; Guallar et al., 2016), especialmente cuando la participación exige un mayor grado de compromiso.

\section{Investigar las audiencias}

Compartimos con Salaverría (2015) la opinión de que la investigación académica debe superar la fase meramente descriptiva y debe ayudar a la innovación. Efectivamente, debe dar un paso adelante hacia la aplicabilidad, debe aportar al sector resultados que ayuden a tomar decisiones y a mejorar.

La investigación debe dar respuestas que sean útiles para el periodismo, pero para ello son necesarias nuevas preguntas. Hay que abandonar los espacios comunes de confort de periodistas y académicos y repreguntarse las propias bases del periodismo, como ¿qué es el periodismo? o ¿qué es noticia hoy? $\mathrm{Y}$, a nuestro entender, estas preguntas deben responderse desde la óptica de la audiencia y no exclusivamente desde la de los profesionales del periodismo. ¿Qué consideran noticia los ciudadanos?, ¿qué es información de calidad para la audiencia?, ¿cómo quieren los ciudadanos relacionarse con los periodistas y la información?

\section{Los números de la audiencia}

La posibilidad de conocer en detalle el comportamiento de la audiencia y cuantificarlo ha conducido a un incremento de su papel en el juicio periodístico, incidiendo en la toma de decisiones de los informadores (Anderson, 2011). Diversas investigaciones han evidenciado la distancia existente entre las preferencias de los periodistas y las de los usuarios (Boczkowski, 2010), y la necesidad de generar tráfico ha motivado que los editores prioricen los intereses de la audiencia (MacGregor, 2007; Van-Dalen, 2012). Como consecuencia de ello, académicos

Figura 2. Opciones para compartir contenidos de El país http://elpais.com/elpais/2016/05/26/ciencia/1464248631_687831.htm 
y profesionales consideran que la poca atención prestada a la conducta de la audiencia, es una de las causas de la progresiva pérdida de calidad periodística (Costera-Meijer, 2013).

Se precisan investigaciones que permitan superar las métricas más burdas centradas en el número de visitas y que aporten información relevante para los medios de modo que éstos puedan alinear su línea editorial y su función democrática con los intereses de los lectores. El número de visitas de una noticia no debe ser traducido como el número de lecturas, ni como las noticias que más valoran los

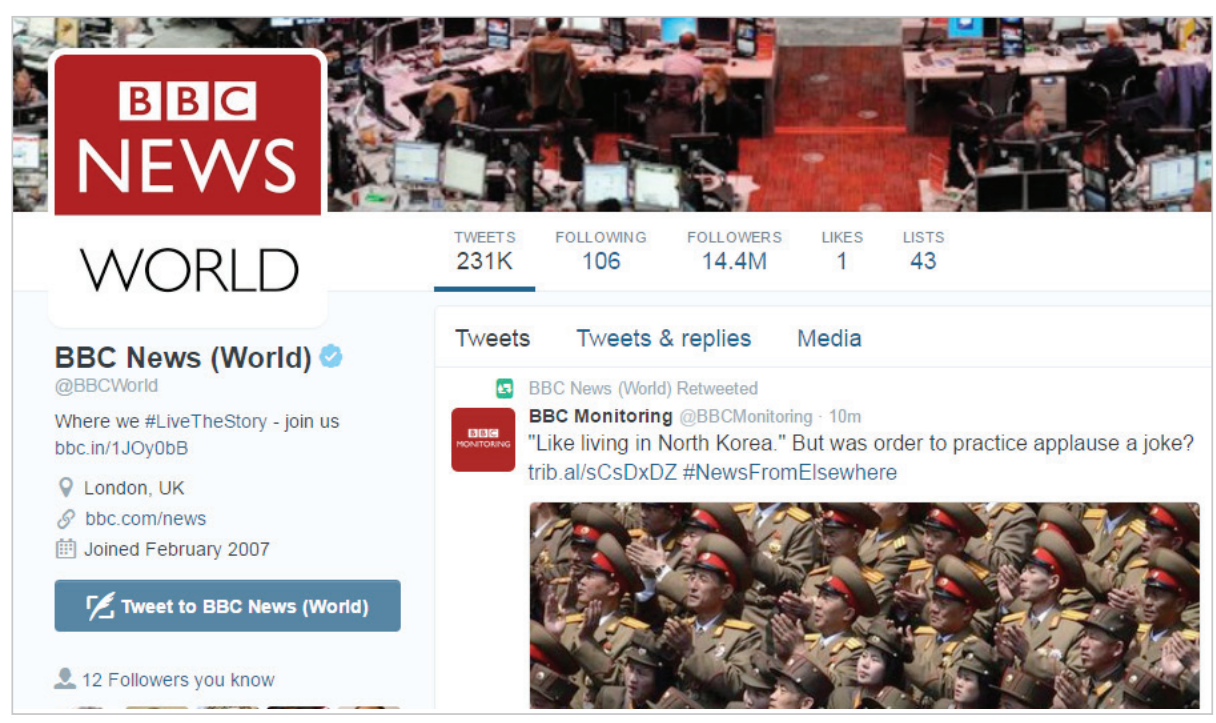

Figura 3. Cuenta de Twitter de la BBC

https://twitter.com/bbc?lang=en lectores, ni de mayor calidad. Las cifras de visitas, comentarios o likes son indicadores de valor de entretenimiento, pero no de periodismo, y mucho menos de buen periodismo (CosteraMeijer, 2013).

\section{La calidad periodística hoy}

Sin duda, abordar el concepto de calidad en periodismo es un reto complejo. El concepto se ha construido desde la óptica profesional, Kümpel y Springer (2015) han identificado cinco dimensiones para definir la calidad periodística: relevancia, exhaustividad, diversidad, imparcialidad y precisión. Diversos autores han denunciado la pérdida de calidad de la información periodística a causa de las presiones económicas y políticas (Picard, 2004; Casero-Ripollés, 2014; Sáez, 2015; Ruiz-Caballero, 2008) y la audiencia así lo percibe (Newman; Levy; Nielsen, 2015). No sólo esas presiones inciden negativamente en la calidad, la "inevitabilidad tecnológica" que se ha apoderado de las redacciones también influye. Bajo el precepto de que todo lo tecnológicamente posible es positivo, los medios han tomado decisiones supuestamente innovadoras que han incidido negativamente en la calidad. La inmediatez es un claro ejemplo de ello. Los medios han competido por ser los primeros en dar una noticia y ello se ha reflejado en la calidad final de los contenidos. La velocidad está reñida con la verificación y el contraste. Esa inevitabilidad tecnológica también rezuma en las nuevas etiquetas asociadas al periodismo: periodismo robot, periodismo drone, incluso periodismo de datos..., donde el foco se pone sobre las herramientas tecnológicas y no en la calidad de los contenidos resultantes.

La velocidad está reñida con la verificación y el contraste

Ante noticias estandarizadas, dirigidas al entretenimiento, y un concepto de calidad en mutación, es preciso preguntarse si habrá ciudadanos que estén dispuestos a pagar por ese tipo de contenidos. Unos contenidos que ya están disponi-

bles en las redes y de forma gratuita. Si el valor del periodismo se mide por likes, visitas y capacidad de entretenimiento, los periodistas tienen poco que decir ante youtubers, instagramers o influencers, que son más y mejores.

Es necesario conocer qué entiende la audiencia por calidad y por qué tipo de contenidos está dispuesta a pagar. Los ciudadanos no renuncian a estar informados, pero hoy por hoy sólo una minoría está dispuesta a pagar y a comprometerse con los medios.

Se introdujeron mecanismos de participación de forma acrítica, asumiendo erróneamente que en la sociedad 2.0 todos querrían participar

\section{Los motivos de la audiencia}

A lo largo de los últimos años los medios han multiplicado los mecanismos de participación de los ciudadanos. Diversas investigaciones (Peters; Witschge, 2015) han evidenciado, sin embargo, que mayores oportunidades de interacción no necesariamente se convierten en mayor compromiso e identificación de la audiencia con el medio.

Bajo la mencionada inevitabilidad tecnológica, los medios han convertido la disponibilidad de herramientas de participación en una finalidad en sí misma. La introducción de mecanismos de participación con lícitos objetivos comerciales se produjo de forma acrítica, asumiendo que en la sociedad 2.0 todo el mundo querría participar. La realidad se ha demostrado mucho más compleja. La introducción de una cultura de la participación es difícil porque implica una nueva forma de relación entre las partes. En el caso de los periodistas el cambio supone una modificación del equilibro de poder entre los actores participantes, de su autoridad y en el proceso de toma de decisiones. Una relación que no parecen estar dispuestos a alterar de manera significativa (Singer et al., 2011). 


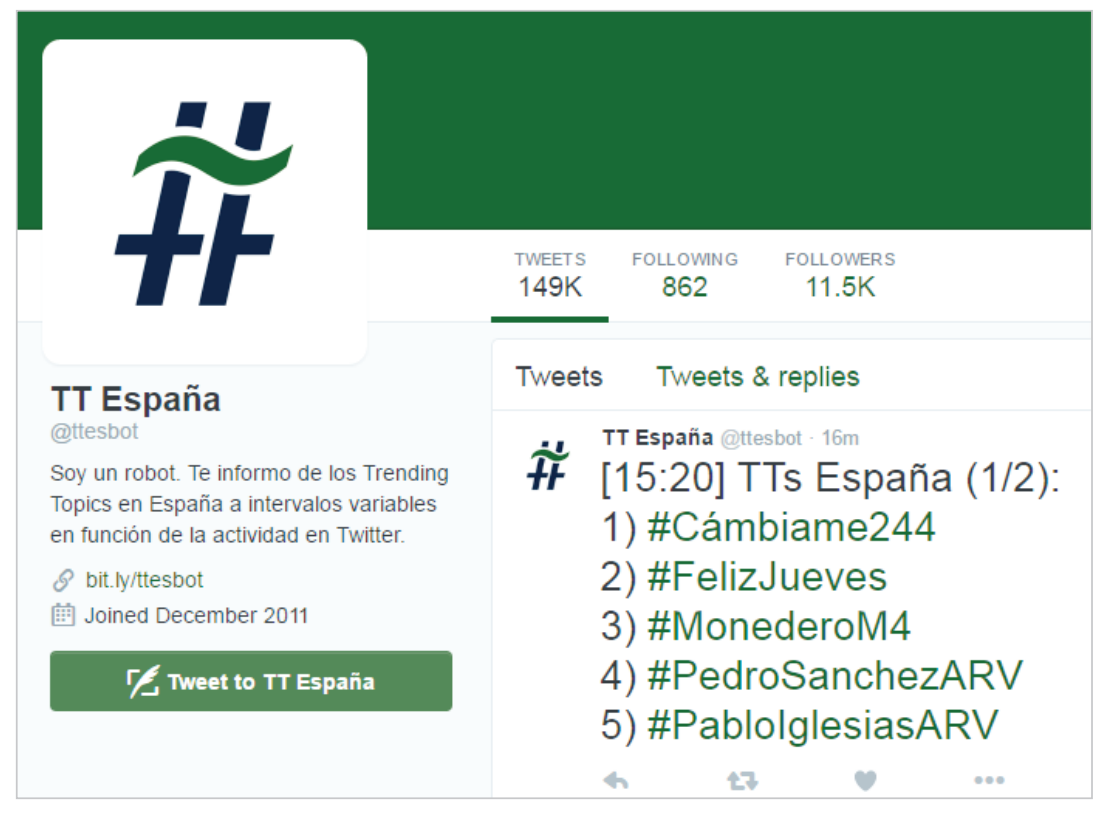

Figura 4. Trending topics en España https://twitter.com/ttesbot

los periodistas información de calidad basada en la verdad y la independencia. Una información que buscan allá donde creen que podrán encontrarla y que después compartirán en las redes sociales.

Las redes sociales se están convirtiendo en los espacios mediáticos donde es más sencillo "encontrar al otro", en espacios donde ciudadanos con distintas visiones del mundo interactúan y donde la información fluye sin los corsés de los medios tradicionales. Las democracias postmodernas necesitan de espacios capaces de facilitar a los ciudadanos el contacto con visiones del mundo que desafían a las propias (Mouffe, 2013). El entendimiento de cómo funcionan estos espacios, de su capacidad de representar una auténtica y funcional esfera pública, es pues de gran importancia para nuestras democracias.

En este sentido ya existen diversas investigaciones que analizan los motivos que impulsan a los ciudadanos a compartir

Pero, por otro lodo, tampoco los ciudadanos parecen demasiado interesados en alterar de manera profunda esa relación de poder entre periodistas y la audiencia. Los ciudadanos no desean hacer de periodistas, "ya existen escuelas para formarlos", y asumen una actitud mucho más modesta, dispuestos a aportar sus experiencias y sus conocimientos al quehacer de los periodistas, pero no para sustituirlos (Borger et al., 2016; Masip et al., 2015; Suau, 2015).

\section{Los ciudadanos aportan experiencias y conocimientos al quehacer de los perio- distas, pero no quieren sustituirlos}

En esta circunstancia falta más información sobre qué motiva a la audiencia a participar, sobre qué espera la audiencia de su interacción con los periodistas. Es preciso profundizar en el conocimiento de qué conduce a los lectores a identificarse con los medios o con un medio determinado. Es bien conocido que se tiende a consumir los medios con los que existe afinidad ideológica (Prior, 2007), sin embargo en el actual ecosistema mediático, ello no es suficiente. Los ciudadanos tienen más opciones donde elegir y son más exigentes. No es suficiente que "su" medio dé una noticia, la Red permite contrastar la información con otros también próximos en la manera de ver el mundo, pero también con aquellos más distantes. La exposición selectiva a la información se diluye y los medios deberían tenerlo en cuenta para recuperar o afianzar la confianza de la audiencia. Los lectores cautivos han desaparecido. Los menos exigentes porque la capacidad de entretenimiento vinculado a la información es compartida por todos los medios y por no profesionales en las redes sociales; y los más exigentes, porque la información-entretenimiento no les satisface y esperan de información a través de las redes sociales, y parecen dibujarse dos grandes conjuntos de motivaciones: la necesidad de afianzar la pertenencia a una comunidad y la autoafirmación personal (Picone; De-Wolf; Robijt, 2016). Es preciso ahondar en esta dirección.

\section{La nueva esfera pública digital}

En la nueva esfera pública digital la audiencia adopta un protagonismo pleno en el proceso informativo. No como productor, no es su pretensión, pero sí como fuente. Además aspira a poder colaborar con los periodistas a los que reconoce su función como narradores de la realidad, y se convierte en gatekeeper en cuanto son los usuarios los que determinan el interés de una noticia y la conveniencia de darle visibilidad a través de un tweet o un me gusta. Falta poder dilucidar si periodistas y usuarios serán capaces de construir comunidad y los primeros convertirse en catalizadores de esa esfera pública de intercambio y debate. Hasta la fecha los resultados no han sido positivos. Los espacios que fueron llamados a desarrollar ese rol, los comentarios, han fracasado rotundamente. A pesar de su éxito en términos cuantitativos, los usuarios que buscan un espacio de discusión a partir de los temas de actualidad, huyen de ellos desencantados ante la falta de respeto no sólo a lo que se dice, sino a quién lo dice. La disparidad es sofocada con menosprecio y descalificaciones (Paskin, 2010; Rowe, 2014; Springer; Engelmann; Pfaffinger, 2015; Ruiz-Caballero et al., 2012). Es necesario determinar si los medios pueden recuperar ese papel de impulsores del debate público o pierden definitivamente esa oportunidad, que se traslada hacia otros espacios, tanto físicos, como digitales. Entre éstos, las redes sociales están aparentemente logrando derivar parte del debate que abandonó los espacios de comentarios de los medios digitales (Suau, 2015; Guallar et al., 2016). Además, no sólo se convierten en espacio para el debate, 
sino que facilitan la reconfiguración de la agenda mediática (Goode, 2009). En tal caso es relevante determinar si la participación de la audiencia en las redes sociales está influida por la agenda mediática; y viceversa, identificar si la agenda de las redes sociales incide en la agenda mediática.

Tras todas estas investigaciones que se han citado existe un común denominador: alcanzar un mejor conocimiento de la audiencia y de su relación con los periodistas y la información. A partir de ese conocimiento es posible establecer una nueva relación entre ambos, una relación más próxima a lo que Lewis y sus colegas denominan periodismo recíproco (Lewis; Holton; Coddington, 2013; Holton; Lewis; Coddington, 2016). Esto es, cómo periodistas y audiencia pueden desarrollar relaciones recíprocas beneficiosas para todos. No en balde, las experiencias periodísticas más satisfactorias surgidas en los últimos años en España se basan en una estrecha relación de reconocimiento mutuo entre periodistas y su audiencia. Y curiosamente, ninguna ha surgido de los medios denominados mainstream.

Las experiencias periodísticas más satisfactorias se basan en una estrecha relación de reconocimiento mutuo entre periodistas y su audiencia

\section{Notas}

1. Este trabajo forma parte de los proyectos de investigación CSO2012-39518-C04-01 y CSO2015-64955-C4-1-R, financiados por el Ministerio de Economía y Competitividad de España.

2. Según el Informe anual de la profesión periodística, desde 2008 la destrucción de empleo en el sector de los medios de comunciación es España se estima en unos 12.200 puestos de trabajo (APM, 2016).

3. Sobre este aspecto resulta revelador el análisis realizado por Francesc Pujol sobre la reacción inicial de los medios españoles ante las filtraciones de los papeles de Panamá. De los 15 medios analizados, 5 no citaron ni a El confidencial, ni a La sexta, a pesar de ser los dos medios españoles que trabajaron en la filtración; y sólo 3 incluyeron enlaces directos a las historias publicadas por esos dos medios:

https://smreputationmetrics.wordpress.com/2016/04/04/ papeles-de-panama-la-reaccion-inicial-de-la-prensa-espanola

\section{Referencias}

Anderson, Christopher W. (2011a). "Between creative and quantified audiences: Web metrics and changing patterns of newswork in local US newsrooms". Journalism, v. 12, n. 5, pp. 550-566.

http://dx.doi.org/10.1177/1464884911402451

Anderson, Christopher W. (2011b). “Deliberative, agonistic, and algorithmic audiences: Journalism's vision of its public in an age of audience transparency". International journal of communication, v. 5, pp. 529-547.

http://ijoc.org/index.php/ijoc/article/viewFile/884/537
APM (2016). Informe anual de la profesión periodística. Asociación de la Prensa de Madrid.

http://www.apmadrid.es/publicaciones/informe-anual-dela-profesion-periodistica

Bergström, Annika (2008). "The reluctant audience: online participation in the Swedish journalistic context". Westminster papers in communication and culture, v. $5, \mathrm{n}$. 2, pp. 60-80.

http://www.westminsterpapers.org/articles/abstract/10.16997/ wpcc. 67

Borger, Merel; Van-Hoof, Anita; Costera-Meijer, Irene; Sanders, José (2013). "Constructing participatory journalism as a scholarly object". Digital journalism, v. 1, n. 1, pp. 117-134.

http://dx.doi.org/10.1080/21670811.2012.740267

Borger, Merel; Van Hoof, Anita; Sanders, José (2016). “Expecting reciprocity: Towards a model of the participants' perspective on participatory journalism". New media \& society, v. 18, n. 5, pp 708-725.

http://dx.doi.org/10.1177/1461444814545842

Boczkowski, Pablo J. (2010). News at work: Imitation in an age of information abundance. Chicago: University of Chicago Press. ISBN: 9780226062785

Boczkowski, Pablo J. (2013). The news gap: When the information preferences of the media and the public diverge. Cambridge, Massachusetts: The MIT Press. ISBN: 978 0262019835

Bruns, Axel (2005). Gatewatching: Collaborative online news production. New York: Peter Lang. ISBN: 0820474320

Casero-Ripollés, Andreu (2014). "La pérdida de valor de la información periodística: causas y consecuencias". Anuario ThinkEPI, v. 8, pp. 256-259.

http://recyt.fecyt.es/index.php/ThinkEPI/article/view/29589

Costera-Meijer, Irene (2013). "Valuable journalism: the search for quality from the vantage point of the user". Journalism, v. 14, n. 6, pp. 754-770.

http://dx.doi.org/10.1177/1464884912455899

Curran James; Fenton, Natalie; Freedman, Des (2012). Misunderstanding the internet. New York: Routledge. ISBN: 9780415579582

Chadwick, Andrew (2013). The hybrid media system: Politics and power. Oxford: Oxford University Press. ISBN: 9780199759484

Fenton, Natalie (2010). New media, old news: journalism and democracy in the digital age. London: Sage Publications. ISBN: 9781847875747

García-De-Torres, Elvira; Yezers'Ka, Lyudmyla; Rost, Alejandro; Calderín, Mabel; Edo, Concha; Rojano, Miladys; Said-Hung, Elías; Jerónimo, Pedro; Arcila, Carlos; SerranoTellería, Ana; Sánchez-Badillo, Jorge; Corredoira, Loreto (2011). "Uso de Twitter y Facebook por los medios iberoamericanos". El profesional de la información, v. 20, n. 6, pp. 611-620.

http://recyt.fecyt.es/index.php/EPI/article/view/epi.2011.nov.02 
Gillmor, Dan (2004). We the media. Grassroots journalism by the people, for the people. Sebastopol, USA: O'Reilly. ISBN: 9780596102272

Goode, Luke. 2009. "Social news, citizen journalism and democracy". New media \& society, v. 11, n. 8, pp. 1287-1305. http://dx.doi.org/10.1177/1461444809341393

Guallar, Javier; Suau, Jaume; Ruiz-Caballero, Carlos; Sáez, Albert; Masip, Pere (2016). “News' redissemination and public debate on social networks". El profesional de la información, v. 25, n. 3, pp. 358-366.

http://dx.doi.org/10.3145/epi.2016.may.05

Harrison, Jackie (2010). "User-generated content and gatekeeping at the BBC hub". Journalism studies, v. 11, n. 2, pp. 243-256.

http://dx.doi.org/10.1080/14616700903290593

Heise, Nele; Loosen, Wiebke; Reimer, Julius; Schmidt, JanHinrik (2013). "Including the audience: Comparing the attitudes and expectations of journalists and users towards participation in German TV news journalism". Journalism studies, v. 15, n. 4, pp. 411-430.

http://dx.doi.org/10.1080/1461670X.2013.831232

Hermida, Alfred; Thurman, Neil (2008). "A clash of cultures: The integration of user-generated content within professional journalistic frameworks at British newspaper websites". Journalism practice, v. 2, n. 3, pp. 343-356. http://dx.doi.org/10.1080/17512780802054538

Holton, Avery E.; Lewis, Seth C.; Coddington, Mark (2016). "Interacting with audiences". Journalism studies, Onlinefirst. http://dx.doi.org/10.1080/1461670X.2016.1165139

Jarvis, Jeff (2015). El fin de los medios de comunicación de masas. Barcelona: Gestión 2000, 222 p. ISBN: 978849875 4018

Jenkins, Henry; Carpentier, Nico (2013). "Theorizing participatory intensities: A conversation about participation and politics". Convergence: The international journal of research into new media technologies, v. 19, n. 3, pp. 265-286.

http://dx.doi.org/10.1177/1354856513482090

Karlsson, Michael; Clerwall, Christer; Nord, Lars (2015). “Please guard the gates, or whatever. Citizens' view on participatory journalism". In: The Future of Journalism Conference : Risks, Threats and Opportunities. Cardiff 10, 11 September.

Klinger, Ulrike; Svensson, Jakob (2015). "The emergence of network media logic in political communication: A theoretical approach". New media \& society, v. 17, n. 8, pp. 1241-1257. http://dx.doi.org/10.1177/1461444814522952

Kovach, Bill; Rosenstiel, Tom (2003). Los elementos del periodismo. Madrid: El País-Santillana. ISBN: 9788403093829

Kümpel, Anna-Sophie; Springer, Nina (2015). Commenting quality: Effects of user comments on perceptions of journalistic quality. In: The future of journalism. Cardiff.

Lee, Angela M.; Chyi, H. Iris (2013). "When newsworthy is not noteworthy. Examining the value of news from the audience's perspective". Journalism studies, v. 15, n. 6, pp. 807-820.
http://dx.doi.org/10.1080/1461670X.2013.841369

Lewis, Seth C.; Holton, Avery E.; Coddington, Mark (2013). "Reciprocal journalism". Journalism practice, v. 8, n. 2, pp. 229-241.

http://dx.doi.org/10.1080/17512786.2013.859840

MacGregor, Phil (2007). "Tracking the online audience: Metric data start a subtle revolution". Journalism studies, v. 8, n. 2, pp. 280-298.

http://dx.doi.org/10.1080/14616700601148879

Masip, Pere (2014). "Audiencias activas, democracia y algoritmos". Anuario ThinkEPI, v. 8, pp. 260-263.

http://recyt.fecyt.es/index.php/ThinkEPI/article/view/29590

Masip, Pere; Guallar, Javier; Suau, Jaume; Ruiz-Caballero, Carlos; Peralta, Miquel (2015). "News and social networks: audience behavior". El profesional de la información, v. 24, n. 4, pp. 363-370.

http://dx.doi.org/10.3145/epi.2015.jul.02

Mouffe, Chantal (2013). Agonistics: Thinking the world politically. London: Verso, 228 pp. ISBN: 9781781681039

Netzer, Yakov; Tenenboim-Weinblatt, Karen; Shifman, Limor (2014). The construction of participation in news websites. Journalism studies, v. 15, n. 5.

http://dx.doi.org/10.1080/1461670X.2014.895527

Newman, Nic; Levy, David A. L.; Nielsen, Rasmus-Kleis (2015). Reuters Institute digital news report.

https://goo.gl/7KgYYb

Nerone, John (2009). "The death (and rebirth?) of workingclass journalism". Journalism, v. 10, n. 3, pp. 353-355. http://dx.doi.org/10.1177/1464884909102596

Paskin, Danny (2010). "Say what? An analysis of reader comments in bestselling American newspapers". Journal of international communication, v. 16, n. 2, pp. 67-83. http://dx.doi.org/10.1080/13216597.2010.9674769

Peters, Chris; Witschge, Tamara (2015). "From grand narratives of democracy to small expectations of participation". Journalism practice, v. 9, n. 1. http://dx.doi.org/10.1080/17512786.2014.928455

Phillips, Angela (2011). "Journalists as unwilling sources: Transparency and the new ethics of journalism". In: Franklin, Bob; Carlson, Matt (eds.). Journalists, sources, and credibility: New perspectives. Routledge, pp. 49-60. ISBN: 9780415835039

Picard, Robert G. (2004). "Commercialism and newspaper quality". Newspaper research journal, v. 25, n. 1, pp. 54-65. http://www.robertpicard.net/PDFFiles/commercialismquality. $p d f$

Picone, Ike; De-Wolf, Ralf; Robijt, Sarie (2016). "Who shares what with whom and why?" Digital journalism. Onlinefirst. http://dx.doi.org/10.1080/21670811.2016.1168708

Prior, Markus (2007). Post-broadcast democracy. Cambridge: Cambridge University Press. ISBN: 9780521675338

Purcell, Kristen; Rainie, Lee; Mitchell, Amy; Rosenstiel, Tom; Olmstead, Kenneth (2010). "Understanding the par- 
ticipatory news consumer. How internet and cell phone users have turned news into a social experience". In: Project for excellence in journalism, pp. 1-51. Washington, DC: Pew Research Center.

http://www.pewinternet.org/2010/03/01/understandingthe-participatory-news-consumer-2

Robinson, Sue (2009). "If you had been with us': Mainstream press and citizen journalists jockey for authority over the collective memory of hurricane Katrina". New media and society, v. 11, n. 4, pp. 795-814.

http://dx.doi.org/10.1177/1461444809105353

Rowe, lan (2014). "Civility 2.0: a comparative analysis of incivility in online political discussion". Information, communication \& society, v. 18, n. 2, pp. 121-138.

http://dx.doi.org/10.1080/1369118X.2014.940365

Ruiz-Caballero, Carlos (2008). La agonía del cuarto poder: prensa contra democracia. Barcelona: Editorial Trípodos. ISBN: 9788493536039

Ruiz-Caballero, Carlos; Domingo, David; Micó, Josep-Lluís; Díaz-Noci, Javier; Meso, Koldo; Masip, Pere (2011). "Public sphere 2.0? The democratic qualities of citizen debates in online newspapers". The international journal of press/politics, v. 16, n. 4.

http://dx.doi.org/10.1177/1940161211415849

Sabadin, Vittorio (2007). The last issue of The New York times: the future of newspapers. Barcelona: Sol90. ISBN: 9788498207736

Sáez, Albert (2015). El periodisme després de Twitter: notes per a repensar un ofici. València: Tres i Quatre. ISBN: 978 8475029665

Salaverría, Ramón (2015). “Ideas para renovar la investigación sobre medios digitales". El profesional de la información, v. 24, n. 3, pp. 223-226.

http://dx.doi.org/10.3145/epi.2015.may.01
Singer, Jane B. (2010). "Quality control: Perceived effects of user-generated content on newsroom norms, values and routines". Journalism practice, v. 4, n. 2, pp. 127-142 http://dx.doi.org/10.1080/17512780903391979

Singer, Jane B.; Domingo, David; Heinonen, Ari; Hermida, Alfred; Paulussen, Steve; Quandt, Thorsten; Reich, Zvi; Vujnovic, Marina (2011). Participatory journalism: guarding open gates at online newspapers. Oxford: Wiley-Blackwell. ISBN: 9781444332261

Springer, Nina; Engelmann, Ines; Pfaffinger, Christian (2015). "User comments: motives and inhibitors to write and read". Information, communication \& society, v. 18, n. 7, pp. 798-815. http://dx.doi.org/10.1080/1369118X.2014.997268

Suau, Jaume (2015). Citizens and online media participation: attitudes and motivations towards participatory journalism. Barcelona: Universitat Ramon Llull. Tesis doctoral. http://www.tesisenred.net/handle/10803/289347

Van-Dalen, Arjen (2012). "The algorithms behind the headlines". Journalism practice, v. 6, n. 5-6, pp. 648-658. http://dx.doi.org/10.1080/17512786.2012.667268

Van-Dijk, José (2009). "Users like you?: theorizing agency in user-generated content". Media, culture and society, v. 31, n. 1, pp. 41-58.

http://dx.doi.org/10.1177/0163443708098245

Vujnovic, Marina; Singer, Jane B.; Paulussen, Steve; Heinonen, Ari; Reich, Zvi; Quandt, Thorsten; Hermida, Alfred; Domingo, David (2010). "Exploring the political-economic factors of participatory journalism". Journalism practice, v. 4, n. 3, pp. 285-296.

http://dx.doi.org/10.1080/17512781003640588

Ward, Stephen J. A. (2005). "Philosophical foundations for global journalism ethics". Journal of mass media ethics, $v$. 20, n. 1, pp. 3-21.

http://dx.doi.org/10.1207/s15327728jmme2001_2

\section{RecBib}

\section{Información sobre empleos, cursos, oposiciones,} noticias, eventos y más cosas del mundo de las bibliotecas, archivos, documentación e información ¡Descúbrenos! 